\title{
Congenital Jejunal Diverticular Bleeding in a Young Adult
}

\author{
Ji-Yung Lee ${ }^{1}$, Jae-Young Jang ${ }^{1}$, Min-Je Kim² ${ }^{2}$ Tae-In Lee ${ }^{2}$, Jung-Wook Kim ${ }^{1}$ and Young-Woon Chang ${ }^{1}$ \\ ${ }^{1}$ Department of Internal Medicine, College of Medicine, Kyung Hee University, Seoul, ${ }^{2}$ Department of medicine, Graduate school, Kyung Hee \\ University, Seoul, Korea
}

Diverticular bleeding of the small bowel is rare and occurs primarily in adults aged more than 60 years. In younger adults, Meckel's diverticulum, a true diverticulum that congenitally occurs in the distal ileum, is the most common cause of diverticular bleeding of the small bowel. Unlike Meckel's diverticula, other kinds of small bowel diverticula are not congenital and their incidence is related to age. Furthermore, congenital true diverticular bleeding of the jejunum in adults is very rare. We report the case of a 24-year-old man with subepithelial tumor-like lesion accompanied with obscure overt gastrointestinal bleeding. This lesion was initially suspected to be a subepithelial tumor based on radiologic tests and capsule endoscopy. He was finally diagnosed with a congenital true diverticulum in the jejunum with the appearance of a Meckel's diverticulum after surgical resection. Clin Endosc 2017;50:495-499

Key Words: Gastrointestinal hemorrhage; Meckel diverticulum; Neoplasms, glandular and epithelial; Jejunum

\section{INTRODUCTION}

Obscure overt gastrointestinal bleeding (OOGIB) accounts for approximately $5 \%$ of all gastrointestinal bleeding. ${ }^{1}$ In approximately $75 \%$ of patients with OOGIB, lesions can be found in the small bowel. ${ }^{2}$ While younger patients are prone to small intestinal tumors, Meckel's diverticula, Dieulafoy's lesions, and Crohn's disease, patients older than 40 years of age are prone to bleeding from vascular lesions (up to $40 \%$ among all causes) and nonsteroidal anti-inflammatory drug-induced small bowel disease. ${ }^{3}$ Among them, diverticular bleeding is very rare and primarily occurs in adults older than 60 years of age. ${ }^{4}$ Histologically, bleeding usually involves false diverticula, with duodenal diverticula accounting for $79 \%$ of cases, and jejunal and ileal diverticula accounting for $18 \%{ }^{5}$ Meckel's diverticulum, which occurs mostly in younger adults, is mainly

Received: November 12, 2016 Revised: March 1, 2017

Accepted: April 12, 2017

Correspondence: Jae-Young Jang

Department of Internal Medicine, College of Medicine, Kyung Hee University, 23 Kyungheedae-ro, Dongdaemun-gu, Seoul 02447, Korea

Tel: +82-2-958-8150, Fax: +82-2-958-8199, E-mail: jyjang@khu.ac.kr

(cc) This is an Open Access article distributed under the terms of the Creative Commons Attribution Non-Commercial License (http://creativecommons.org/ licenses/by-nc/3.0) which permits unrestricted non-commercial use, distribution, and reproduction in any medium, provided the original work is properly cited. true diverticulum that occurs in the distal ileum. ${ }^{6}$ It is usually located on the anti-mesenteric border of the ileum within about $60 \mathrm{~cm}$ of the terminal ileum. ${ }^{7}$ In South Korea, there are no reports of congenital true diverticular bleeding of the jejunum in adults. Herein, we report the case of a young adult with OOGIB, who was diagnosed with a congenital true diverticulum in the jejunum (with the appearance of a Meckel's diverticulum), after being initially mistaken for a subepithelial tumor of the small intestine.

\section{CASE REPORT}

A 24-year-old man with no specific history visited our hospital presenting with melena, which had developed approximately one week earlier, and hematochezia, which had started two days before. Gastroscopy and colonoscopy performed at another hospital did not reveal the cause; therefore, he visited our hospital for a closer inspection.

At the time of the visit, his blood pressure was $124 / 80 \mathrm{~mm}$ $\mathrm{Hg}$, and he had a pulse of 84 beats per minute, a breathing rate of 20 breaths per minute, and a body temperature of $36.6^{\circ} \mathrm{C}$. Melena was observed in a digital rectal examination. No palpable mass in the abdomen, tenderness, or rebound tenderness were found. The results of a peripheral blood test 

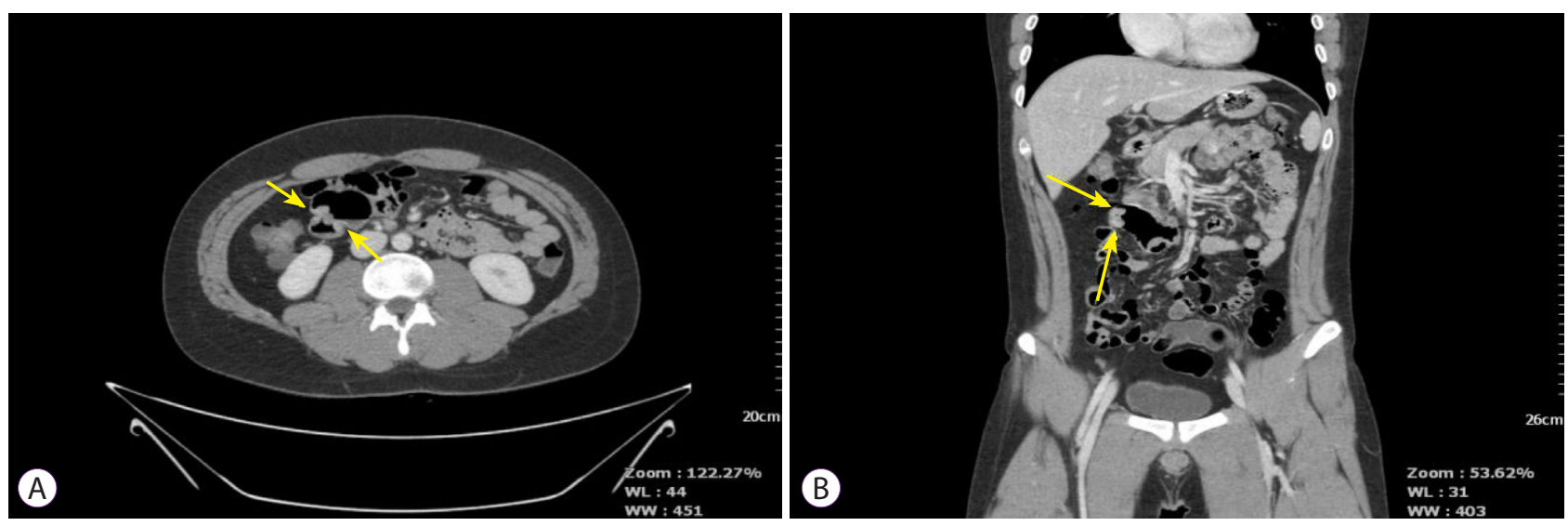

Fig. 1. Abdominal computed tomography. (A) The axial view shows a pouch or mass-like lesion of the small bowel in the right middle abdomen with thickening of the bowel wall. (B) In the coronal view, the yellow arrow indicates circumferential wall thickening of the jejunal loop in the right middle abdomen and dilatation of the proximal area.
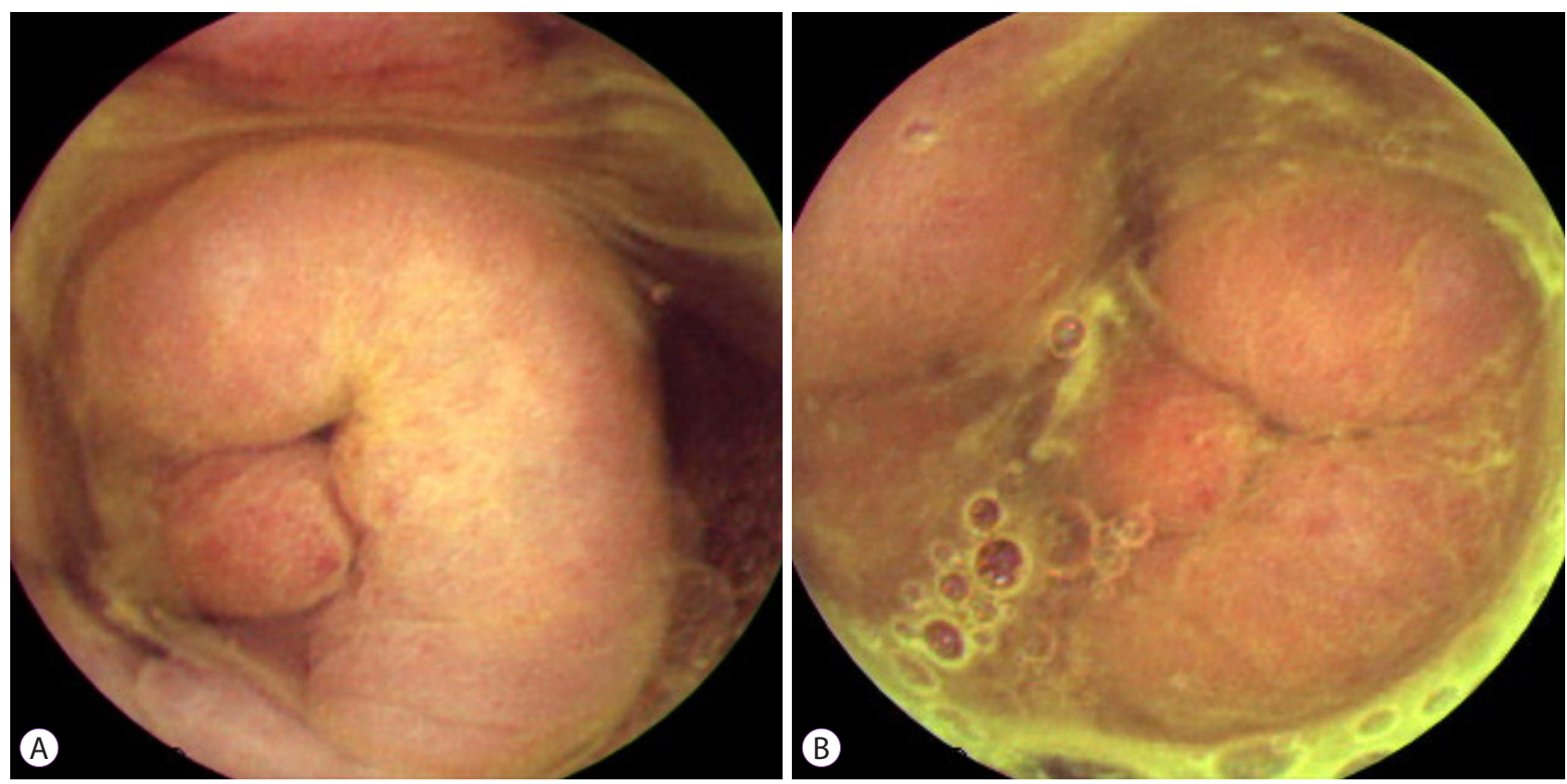

Fig. 2. (A, B) Capsule endoscopy showing a mass-like lesion at the jejunum without mucosal changes suggesting ulcer or erosion. The consistency of the lesion was uncertain. Finally, after surgical resection, this lesion was confirmed to be a mucosal hypertrophy with diverticular orifice.

performed the day before at another hospital were as follows: white blood cell count, 5,300/ $\mathrm{mm}^{3}$; hemoglobin, $12.1 \mathrm{~g} / \mathrm{dL}$; hematocrit, $37 \%$; and blood platelet count, $318,000 / \mathrm{mm}^{3}$. Serum biochemical test results were as follows: total protein, $7.3 \mathrm{~g} / \mathrm{dL}$; albumin, $4.3 \mathrm{~g} / \mathrm{dL}$; total bilirubin, $0.8 \mathrm{mg} / \mathrm{dL}$; aspartate aminotransferase (AST), $22 \mathrm{IU} / \mathrm{L}$; alanine aminotransferase (ALT), $16 \mathrm{IU} / \mathrm{L}$; blood urea nitrogen concentration, $9.9 \mathrm{mg} / \mathrm{dL}$; and creatinine, $1 \mathrm{mg} / \mathrm{dL}$.

Abdominal computed tomography was performed. A subepithelial tumor was suspected due to a circular thickening of the intestinal wall without mucosal enhancement or defect at the estimated position of the distal jejunal loop in the right abdomen. Although the proximal area of the lesion was mild- ly dilated, there was no definite sign of intestinal obstruction (Fig. 1). Wireless capsule endoscopy (WCE) was performed to determine whether this finding reflected a significant lesion or was the result of normal peristalsis. The capsule endoscopy showed a lesion resembling a tumor in the distal area of the jejunum (Fig. 2, Supplementary Video 1). There was no change in the mucous membrane, and there was a delay of 4.5 hours in the passage of the capsule through the location of the finding. It was difficult to investigate the firmness of the lesion, and ulcers or erosion could not be clearly observed; however, the lesion was assumed to be the cause of the bleeding. Given this assumption, a small bowel wedge resection was performed for a mass-like lesion with bleeding. Laparoto- 

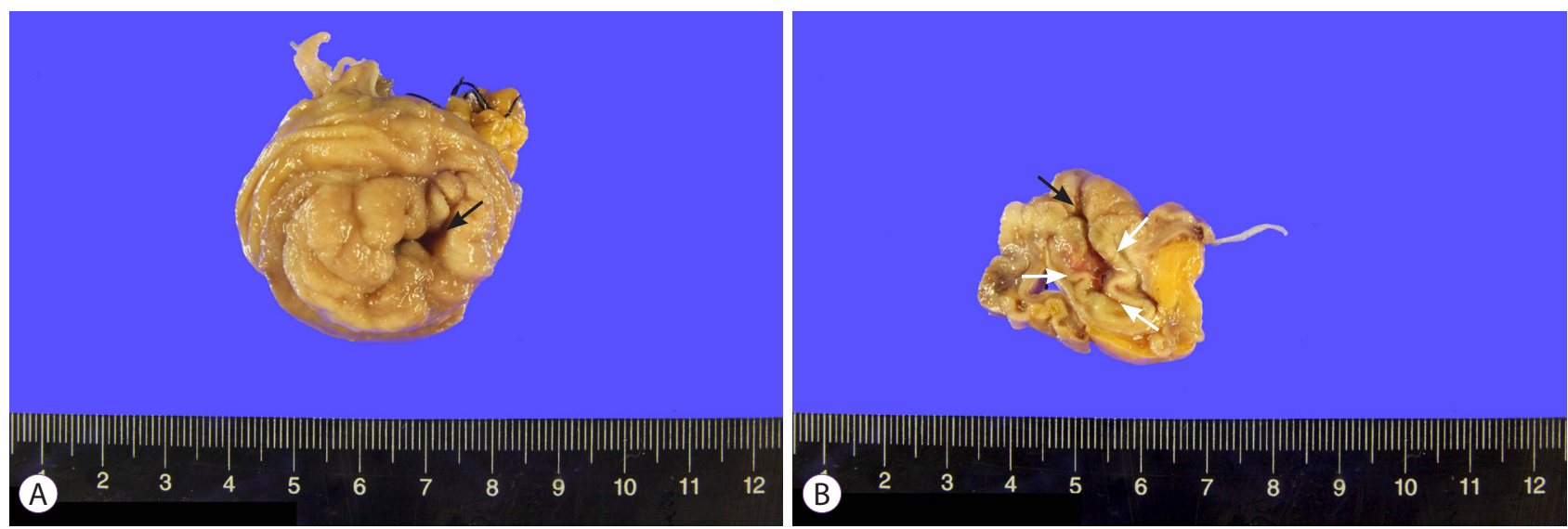

Fig. 3. Macroscopic examination. (A) The orifice of the blind pouch in the jejunum (black arrow) is surrounded by irregular elevated mucosa. (B) A cut section shows that the blind pouch had a muscular layer (the black arrow indicates the orifice and the diverticula pouch were demarcated by white arrows).
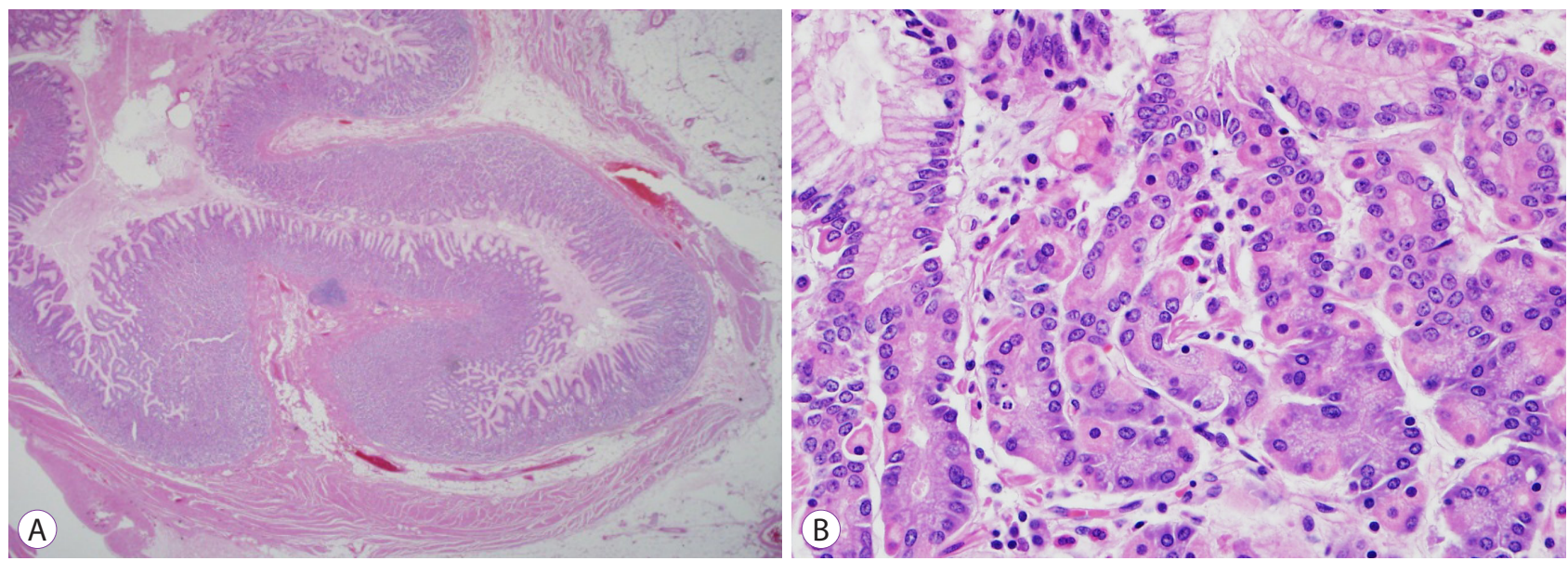

Fig. 4. Microscopic examination. (A) Microscopically, the blind pouch is revealed to be a true diverticulum with a muscular layer (H\&E stain, $\times 12.5)$. (B) Diverticulum is lined by gastric oxyntic mucosa (H\&E stain, $\times 400)$.

my confirmed the presence of a $5 \mathrm{~cm}$ diverticula on the distal jejunal portion. The treatment involved the resection of a 3.5 $\mathrm{cm}$ jejunal segment, removing all visible diverticula. End-toend anastomosis was performed.

Macroscopically, the resected segment of the jejunum had a blind pouch, measuring approximately $2.0 \mathrm{~cm}$ in length and $0.8 \mathrm{~cm}$ in diameter (Fig. 3A), with mucosal elevation around the orifice (Fig. 3B). On microscopic examination, the blind pouch was revealed to be a true diverticulum showing a muscular layer (Fig. 4A) and lined by gastric oxyntic mucosa consisting of parietal, chief, and foveolar epithelial cells (Fig. $4 \mathrm{~B})$. Therefore, the pathologic diagnosis was congenital diverticulum with gastric heterotopia.

The patient was discharged 4 days after the operation without any specific symptoms, and there has been no symptom recurrence for 1 year.

\section{DISCUSSION}

OOGIB is defined as repetitive or persistent gastrointestinal bleeding, for which the cause cannot be determined via upper-gastrointestinal endoscopy and colonoscopy. Intestinal diverticula as a cause of OOGIB accounts for only $5 \%-15 \%$ of cases. ${ }^{8}$ In this case, a young adult with no specific history had melena and hematochezia for more than 3 days, for which the cause and location could not be determined via endoscopy. The lesion observed on an abdominal computed tomography scan and capsule endoscopy was suspected to be a jejunal subepithelial tumor, but was finally determined to be a jejunal diverticulum following surgery.

Intestinal diverticula occur mostly in patients aged 60 years or older, with only $20 \%$ occurring in patients younger than 40 years of age. ${ }^{9}$ A Meckel diverticulum, which is the most common type of congenital diverticulum found in young adults, is a vestigial remnant of an incomplete closure of the 
omphalomesenteric duct, and is the most frequently observed congenital gastrointestinal deformation. ${ }^{6}$ According to the location of the diverticulum, the mean distance from the ileo-cecal valve seems to vary with age. ${ }^{10}$ Arnold et al. demonstrated that the average distance was $34 \mathrm{~cm}$ in children under 2 years of age, $46 \mathrm{~cm}$ in people aged 3-21 years, and $67 \mathrm{~cm}$ in adults. ${ }^{10}$ Histologically, congenital diverticula are true diverticula that consist of all layers of the intestinal wall, including the muscular layer, unlike false diverticula, which occur when only the mucous membrane and submucosa escape through a weak portion of the intestinal wall. Ectopic mucosa, such as gastric or pancreatic mucosa, is often seen in symptomatic patients ${ }^{6,11}$ and can be associated with bleeding. ${ }^{10}$ Our case involved a true diverticulum, surrounded by a muscular layer and including a significant amount of ectopic gastric mucosa and villus cells of the intestine. Furthermore, Meckel's diverticula often show mucosal hypertrophy at the entrance of the diverticulum, as was observed during the capsule endoscopy in the present case, although it initially seemed to be a subepithelial tumor (Fig. 2, Supplementary Video 1). Based on these findings, the case was diagnosed as a congenital diverticulum of the jejunum with the appearance of a Meckel's diverticulum.

Most Meckel's diverticula are asymptomatic and are discovered incidentally in radiological tests. However, complications can occur, including chronic gastrointestinal disorders (abdominal pain, diarrhea, and malabsorption). Serious complications occur in approximately $4 \%$ of cases, and include gastrointestinal bleeding or perforation. ${ }^{7,12}$ Complications are more common in children and decrease in frequency with age. Hence, a diagnosis is usually delayed in adults, and the complication-related fatality rate is reported to be in the range of $6 \%-7.5 \%,{ }^{13}$ When symptoms occur, a diagnosis using radiological tests alone is difficult, and the diagnosis rate for obscure intestinal bleeding is very low. ${ }^{14}$ In the present case, WCE was used to diagnose the cause of bleeding. WCE is noninvasive, has a diagnostic rate of $55 \%$, and is considered more useful than previous methods of intestinal contrast imaging and enteroclysis. However, WCE has limitations: other endoscopic treatments such as biopsy or hemostasis are not possible, and the false negative rate remains high. ${ }^{15}$ Complications including closure or massive hemorrhage require a clear diagnosis and treatment via surgery. ${ }^{16}$

Congenital diverticula primarily occur within $100 \mathrm{~cm}$ proximal to the ileocaecal plate, with only $28 \%$ occurring at a distance of more than $100 \mathrm{~cm} .7$ Among cases of congenital diverticula, only a single case involving the jejunum has been previously reported ${ }^{17}$ (a 4-year old male patient with accompanying intestinal perforation). The present case is the first reported case of a congenital diverticulum accompanied with bleeding in the proximal jejunum.

In conclusion, we report a case of a congenital diverticulum located in the proximal jejunum accompanied by gastrointestinal bleeding, which was initially suspected to be a subepithelial tumor based on radiologic tests and endoscopy, but was later confirmed, by histologic examination, to be a congenital true diverticulum with ectopic gastric mucosa. The lesion resembled a Meckel's diverticulum, and owing to its location in the proximal jejunum, is considered a rare case.

\section{Conflicts of Interest}

The authors have no financial conflicts of interest.

\section{Supplementary Material}

Video 1. Capsule endoscopy showing subepithelial tumor-like lesion at the jejunum (https://doi.org/10.5946/ce.2016.154.v001).

\section{REFERENCES}

1. Gralnek IM. Obscure-overt gastrointestinal bleeding. Gastroenterology 2005;128:1424-1430.

2. Tee HP, Kaffes AJ. Non-small-bowel lesions encountered during double-balloon enteroscopy performed for obscure gastrointestinal bleeding. World J Gastroenterol 2010;16:1885-1889.

3. Liu K, Kaffes AJ. Review article: the diagnosis and investigation of obscure gastrointestinal bleeding. Aliment Pharmacol Ther 2011;34:416423.

4. Raju GS, Gerson L, Das A, Lewis B; American Gastroenterological Association. American gastroenterological association (AGA) institute medical position statement on obscure gastrointestinal bleeding. Gastroenterology 2007;133:1694-1696.

5. Albert JG, Lübbert C, Surow A, Zeuzem S. [Small bowel diverticula unknown disease]. Z Gastroenterol 2009;47:674-681.

6. Sumer A, Kemik O, Olmez A, et al. Small bowel obstruction due to mesodiverticular band of Meckel's diverticulum: a case report. Case Rep Med 2010;2010

7. Dumper J, Mackenzie S, Mitchell P, Sutherland F, Quan ML, Mew D. Complications of Meckel's diverticula in adults. Can J Surg 2006;49:353357.

8. Townsend CM Jr, Beauchamp RD, Evers BM, Mattox KL. Sabiston textbook of surgery 20th edition: the biological basis of modern surgical practice. Philadelphia (PA): Elsevier; 2017. 2176p.

9. Peery AF, Barrett PR, Park D, et al. A high-fiber diet does not protect against asymptomatic diverticulosis. Gastroenterology 2012;142:266272.el.

10. Arnold JF, Pellicane JV. Meckel's diverticulum: a ten-year experience. Am Surg 1997;63:354-355.

11. Dutta G, Chowdhury AS, Panda M. Band of cacophony - abdominal catastrophe caused by the fibrous band of Meckel's diverticulum: a case report. Cases J 2009;2:7160.

12. Park JJ, Wolff BG, Tollefson MK, Walsh EE, Larson DR. Meckel diverticulum: the Mayo Clinic experience with 1476 patients (1950-2002). Ann Surg 2005;241:529-533.

13. Kusumoto H, Yoshida M, Takahashi I, Anai H, Maehara Y, Sugimachi K Complications and diagnosis of Meckel's diverticulum in 776 patients. Am J Surg 1992;164:382-383.

14. Gerson LB, Fidler JL, Cave DR, Leighton JA. ACG clinical guideline: diagnosis and management of small bowel bleeding. Am J Gastroenterol 2015;110:1265-1287; quiz 1288.

15. Costamagna G, Shah SK, Riccioni ME, et al. A prospective trial compar- 
ing small bowel radiographs and video capsule endoscopy for suspected small bowel disease. Gastroenterology 2002;123:999-1005.

16. Hartmann D, Schilling D, Bolz G, et al. Capsule endoscopy versus push enteroscopy in patients with occult gastrointestinal bleeding. Z Gastro- enterol 2003;41:377-382.

17. Yong K, Yoo JH, Choi YB. Congenital jejunal diverticulum. Korean J Pathol 1983;17:302-305. 